\title{
Physiology \\ Obesity as a threat to national security: the need for precision engagement
}

\author{
Jameson D. Voss ${ }^{1} \cdot$ Greg Pavela ${ }^{2} \cdot$ Fatima Cody Stanford $\mathbb{C}^{3}$ \\ Received: 12 January 2018 / Revised: 27 January 2018 / Accepted: 9 February 2018 / Published online: 9 March 2018 \\ (c) Macmillan Publishers Limited, part of Springer Nature 2018
}

The social consequences of increasing obesity prevalence are of pressing concern to scholars, public-figures, and policy-makers, several of whom have posited that obesity threatens national security. In this editorial, we consider the legitimacy of this claim and discuss the importance of obesity's potential to threaten security in the United States and around the globe. Finally, we conclude by noting potential harms of framing obesity as a national security threat.

There are no currently accepted standards for what constitutes a health-related threat to national security. A previous editorial argued that the "military importance" of health factors can be measured with four quantifiable components (military operations, medical cost, compassion/modifiability, and political/public concern) [1]. Others have considered security aspects of civilian health, including vulnerability to attack, childhood fitness for future military service [2, 3], and the health of people living in war-prone areas where social instability could lead to violence. Beyond the probability of war or the economic and human resources needed to fight it, populations with worse health will generally have more health-related vulnerabilities to weapons of mass destruction and pandemic disease.

Obesity applies to all these domains (Fig. 1) of national security. In the United States, an estimated $\$ 1.5$ billion in health care expenses and resources to replace unfit

Fatima Cody Stanford

fstanford@mgh.harvard.edu

1 United States Air Force School of Aerospace Medicine, Aerospace Education and Training Division, Wright-Patterson AFB, Dayton, OH 45433, USA

2 Department of Health Behavior, University of Alabama at Birmingham, Birmingham, AL 35294, USA

3 Departments of Internal Medicine- Gastroenterology and Pediatric Endocrinology, Massachusetts General Hospital Weight Center, Harvard Medical School, Boston, MA 02114, USA personnel are attributed to obesity [2]. However, this estimate is uncertain and may be conservative because the health care costs associated with obesity among military personnel have not been quantified since 2007 and do not include long-term disability expenditures paid outside the U.S. military budget through Veterans Affairs. The impact of obesity on a nation's functional capacity for defense may be even more alarming. Lost work days, degraded readiness/resilience, excess need for aeromedical evacuation, and wide scale recruit ineligibility represent more insidious threats. While class II and III obesity modestly predict risk of commercial truck crashes [4], a similar risk in the military (where operating costly, highly lethal equipment is common) could have severe consequences.

The magnitude of the security threat from obesity remains uncertain, given the numerous potential and unanticipated ways in which obesity might affect military personnel and resources required to support them. Future research should assess whether obesity alters an individual's predisposition to conflict, given some evidence of an association between diabetes and G6PD deficiency and aggressiveness, violent crime, war killings, and/or other correlates of violence [5]. Finally, obesity is associated with immunologic dysfunction, so a greater prevalence of obesity may result in greater population vulnerability to security threats. Specifically, the obesity attributed vulnerability to weapons, psychological terror, and naturally occurring pandemic threats should be investigated for all populations.

Previous literature suggests that effective obesity policy requires integration of diverse stakeholders in society, including the government, in order to drive meaningful progress [6]. By considering the argument that obesity poses a national security threat, there is potential to broaden public support as individuals and groups who might not have had a traditional interest in obesity might glean the importance of this disease on their national standing and personal wellbeing. If the goal is a broad appeal for government action on obesity, the problem should be framed in 
Fig. 1 Health Related National Security Threat Assessment

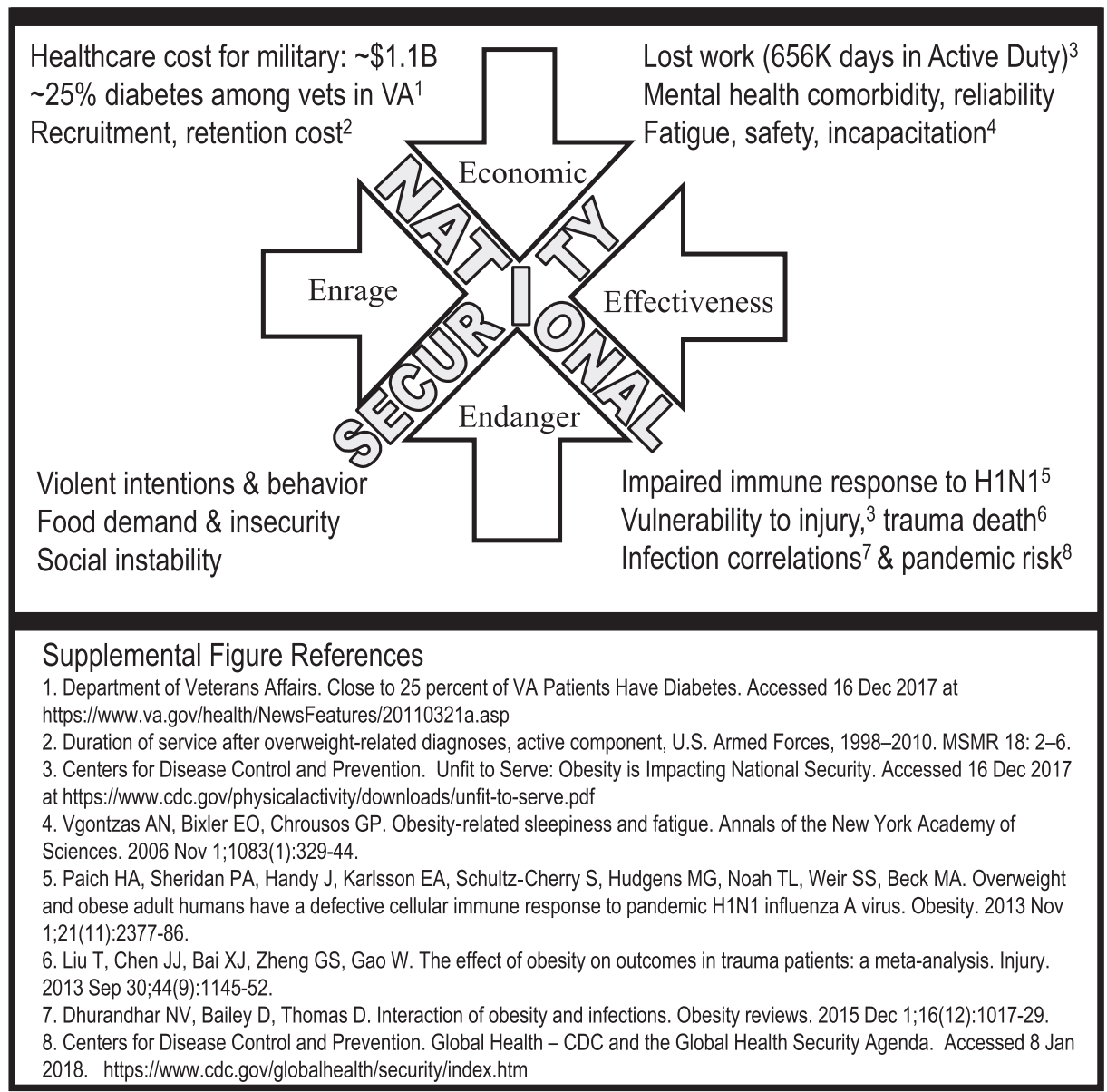

such a way that support is likely regardless of political party affiliation or interest.

A recent study in the United States indicates that, with regards to obesity, Democrats are more persuaded by gainframed messages (i.e., benefits to a society by establishing policies to reduce obesity) than "loss-framed" (i.e., costs to a society that fails to establish those policies), while there was no framing effect on perceived argument strength among Republicans [7]. Over the last 20 years in the United Kingdom, there was a shift in framing obesity from a problem of individual responsibility, towards collective responsibility, and back to the individual in U.K. government reports, policies, and interventions due to the belief that individualistic framing of obesity policy offers the path of least resistance [8].

Beyond public support in general, framing obesity as a national security threat might also alter the public's priorities in other ways. Perhaps support would shift our focus towards the prevention and reduction of obesity in populations who are young and from rural states where accession into the military is more common. Although bariatric surgery is an excellent tool to treat moderate-to-severe obesity, it makes one ineligible to serve in the U.S. military. From a national security lens, prevention of obesity appears more strategic.

While military personnel and planners are obligated to consider potential threats to national security, including the challenges posed by obesity, we wish to emphasize the framing, research, and discussion of obesity's potential effects on national security must be cautious and considered. Obesity stigma is pervasive and has become more common over time[9]. In the United States population, the self-reported experience of weight discrimination is associated with a $60 \%$ higher risk of mortality-generally higher than the risk of mortality associated with discrimination due to race, sex or age [10]. By focusing on the harms of obesity not just to individuals with obesity, but to collective-wellbeing, the stigmatization of obesity may be enhanced. National security and the military hold relatively honored positions in the public opinion. Invoking threats to national security thus has potential benefits from a public health perspective-broadening the coalition of stakeholders interested in addressing obesity-but also has risks, including obesity stigmatization. Thus, scholars should hold 
a precautionary ethos (primum non-nocere) and use precise language.

Specifically, we advise caution before using catastrophizing, hyperbole, puns, and combat language (i.e., "battle of the bulge") which, though intended to communicate public sentiment toward a disease, could be misdirected toward an individual or even suggest a scientific claim. Figure 1 identifies aspects of health-related threats to security that could be quantified, enabling comparisons across diseases. Using available evidence to quantify and qualify the nature of the threat enhances precision, however, population level evidence (e.g., prevalence, burden) tends to rely on observational methods. Thus, it will be important to differentiate correlation and causation, assess the pitfalls of synthesizing disparate data and think critically about inferential errors common in narrative reviews and inductive reasoning.

\section{Disclaimer}

The views expressed in this article are those of the authors and do not necessarily reflect the official policy or position of the Air Force, the Department of Defense, or the U.S. Government. Cleared 88PA, Case Number \# 2018-0140, 11 Jan 2018.

Funding: This work was funded by National Institutes of Health NIDDK R01 DK103946-01A1

\section{Compliance with ethical standards}

Conflict of interest The authors declare that they have no conflict of interest.

\section{References}

1. Armed Forces Health Surveillance Center-AFHSC. "Military importance": what does it mean and can it be assessed objectively? MSMR. 2012;19:2-3.

2. Christeson W, Clifford K, Taggart A. Retreat is not an option: healthier schools meals protect our children and our country. Washington, D.C.: Mission Readiness; 2017. http://missionrea diness.s3.amazonaws.com/wp-content/uploads/MR-NAT-RetreatNot-an-Option.pdf. Accessed 16 Dec 2017.

3. Defense Health Agency/Defense Health Board Falls Church United States. DHB implications of trends in obesity and overweight for the DoD—fit to fight fit for life. 2013. Accession number: AD1027323. https://health.mil/Reference-Center/ Reports/2013/11/22/DHB-Implications-of-Trends-in-Obesity-andOverweight-for-the-DoD-Fit-to-fight-fit-for-life. Accessed 26 Jan 2018.

4. Anderson JE, Govada M, Steffen TK, Thorne CP, Varvarigou V, Kales SN, et al. Obesity is associated with the future risk of heavy truck crashes among newly recruited commercial drivers. Accid Anal Prev. 2012;49:378-84.

5. DeWall CN, Deckman T, Gailliot MT, Bushman BJ. Sweetened blood cools hot tempers: physiological self-control and aggression. Aggress Behav. 2011;37:73-80.

6. Stanford FC, Kyle TK. Why food policy and obesity policy are not synonymous: the need to establish clear obesity policy in the United States. Int J Obes. 2015;39:1667-8.

7. Lee TK, Kim HK. Differential effects of message framing on obesity policy support between democrats and republicans. Health Commun. 2017;32:1481-90.

8. Ulijaszek SJ, McLennan AK. Framing obesity in UK policy from the Blair years, 1997-2015: the persistence of individualistic approaches despite overwhelming evidence of societal and economic factors, and the need for collective responsibility. Obes Rev. 2016;17:397-411.

9. Andreyeva T, Puhl RM, Brownell KD. Changes in perceived weight discrimination among Americans, 1995-1996 through 2004-2006. Obesity (Silver Spring). 2008;16:1129-34.

10. Sutin AR, Stephan Y, Terracciano A. Weight discrimination and risk of mortality. Psychol Sci. 2015;26:1803-11. 